\title{
Supporting Information: Nonlinear wavefront shaping and imaging in nonlinear photonic crystals with a generalized quasi-multivalue-encoding method
}

Ronger Lu, Ruizhi Zhao, Xia Feng, Chao Zhang, ${ }^{*}$ Xuhao Hong, ${ }^{*}$ Yiqiang Qin, ${ }^{*}$ and Yongyuan Zhu

Ronger Lu is with the National Laboratory of Solid State Microstructures, Nanjing University, Nanjing 210093, China and Department of Physics, Nanjing Tech University, Nanjing 211816, China.

Ruizhi Zhao, Xia Feng, Chao Zhang and Yiqiang Qin are with the National Laboratory of Solid State Microstructures, and the College of Engineering and Applied Sciences, Nanjing University, Nanjing 210093, China.

Xuhao Hong and Yongyuan Zhu are with the National Laboratory of Solid State Microstructures, and the School of Physics, Nanjing University, Nanjing 210093, China.

* Corresponding Author: Chao Zhang: zhch@ @ju.edu.cn;

Xuhao Hong: xhhong@nju.edu.cn;

Yiqiang Qin: yqqin@nju.edu.cn;

\section{$\underline{\text { Section I: Comparison among different interval modulations }}$}

Theoretically, binary or any multi-interval modulation can be adopted if there is no fabrication limit. It can be guessed that the more interval modulation, the better the imaging quality, but the higher the fabrication accuracy requirements.

We take the binary, four-interval, six-interval, eleven-interval modulations for comparison to generate the target image of two-focused points with the intensity ratio 4:1, results are exhibited in Figure S1. The intensity of electric field has been normalized. When using the different interval modulations, the corresponding intensities of the weaker point are $0.0800,0.2194,0.2451$ and 0.25 , respectively. As a result, compared with the binary encoding method (intensity ratio 12.5:1), whether it is four-interval (intensity ratio 4.6:1), six-interval (intensity ratio 4.1:1), or eleven-interval (intensity ratio 4:1) modulations, it is much closer to the ideal situation.

The accuracy of binary encoding method to reconstruct the real image is only $32 \%$, while the four-interval modulation is $88 \%$, the six-interval modulation is $98 \%$, and the eleven-interval modulation is approximately $100 \%$. However, limited by fabrication techniques, not all interval modulations can be realized in experiments. The minimum modulation domain structure designed by these methods are $5 \mu \mathrm{m}, 1 \mu \mathrm{m}, 0.5 \mu \mathrm{m}, 0.25$ $\mu m$, respectively. Nowadays, domain structures of sub-micron size are not easily 
achieved by electric field poling at room temperature. So, we have made a trade-off between fabrication limit and imaging quality. Four-interval modulation has been adopted in this paper, which can be achieved in experiments and largely improves the imaging quality compared with the conventional binary encoding method.

(a)

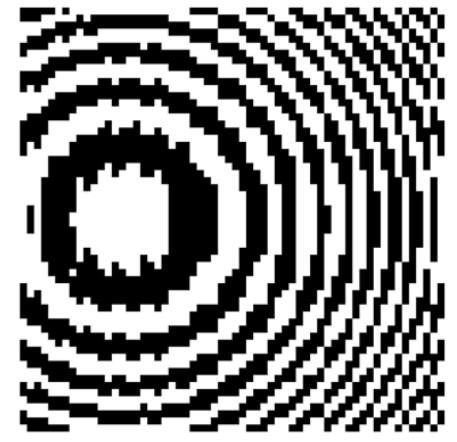

(b)

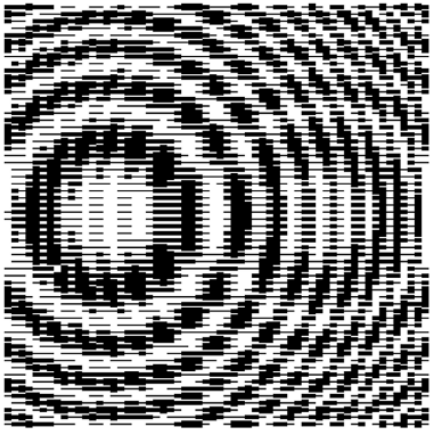

(c)

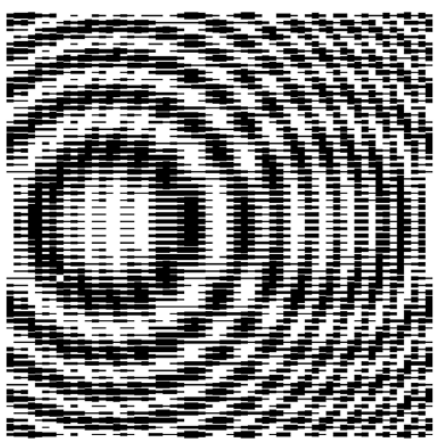

(d)

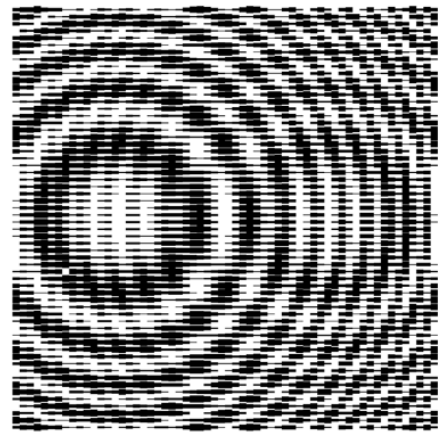

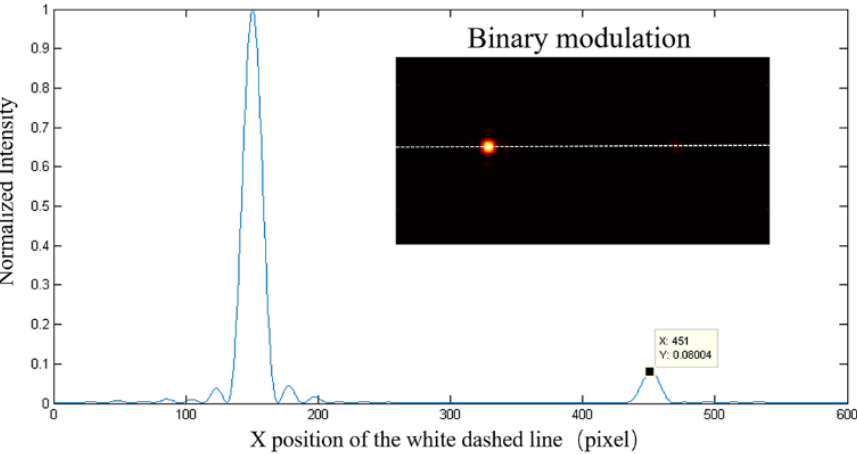
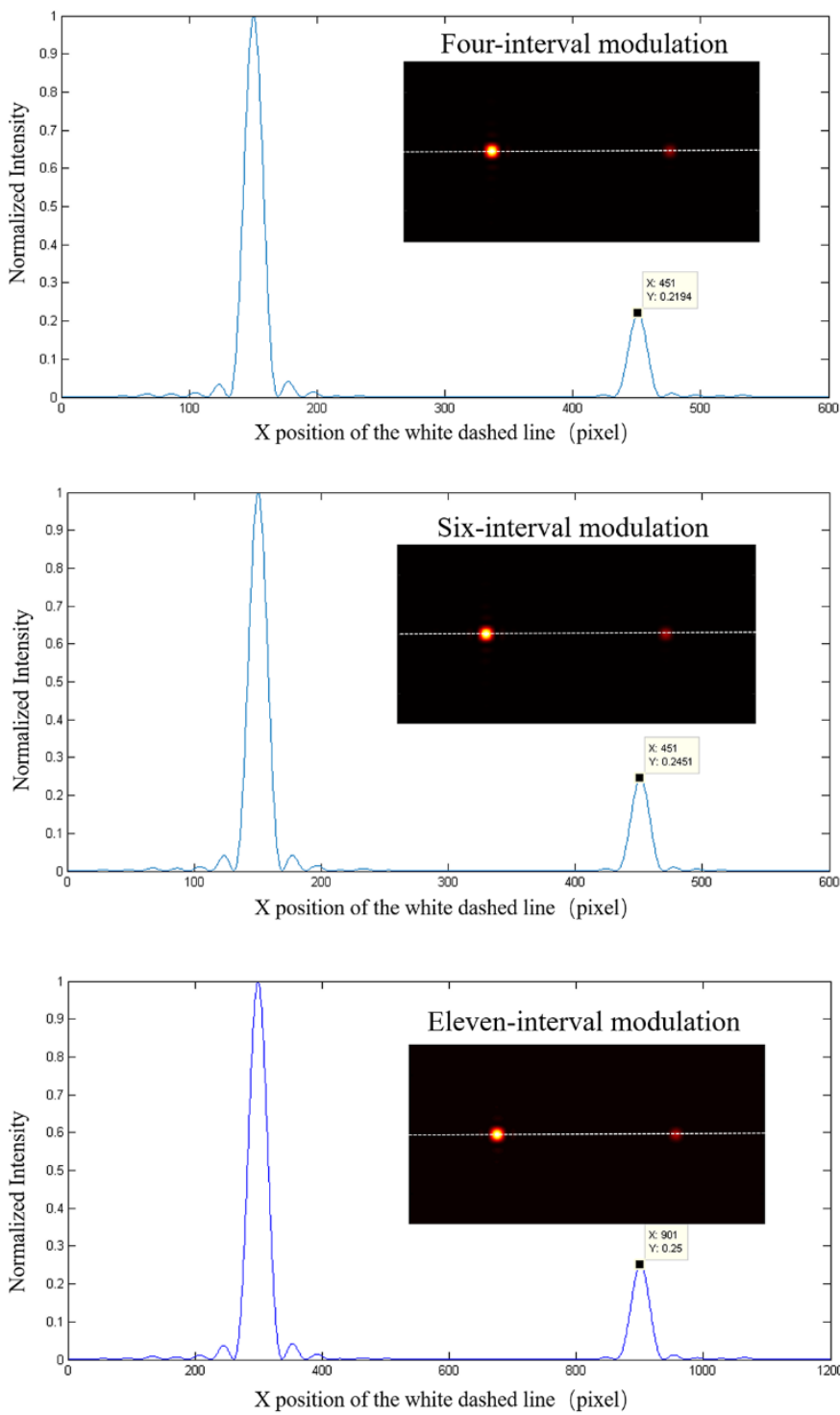

Figure S1 Comparisons among the (a) binary, (b) four-interval, (c) six-interval, (d) eleven-interval modulations to generate the target image of two-focused points with the intensity ratio $4: 1$. 
Section II: Environmental scanning electron microscope (ESEM) images of the structures

Figure S2 shows the corresponding 2D NPC structures designed by binary encoding and the QME method, respectively. The domain images are obtained by the ESEM, with the image conditions being voltage $30 \mathrm{kV}$, pressure $30 \mathrm{~Pa}$, spots 6 , scan rate 30 $\mu \mathrm{s} /$ frame. The white and black regions represent positive domain and negative domain respectively. The thin lines on the picture may be scratches left by polishing.
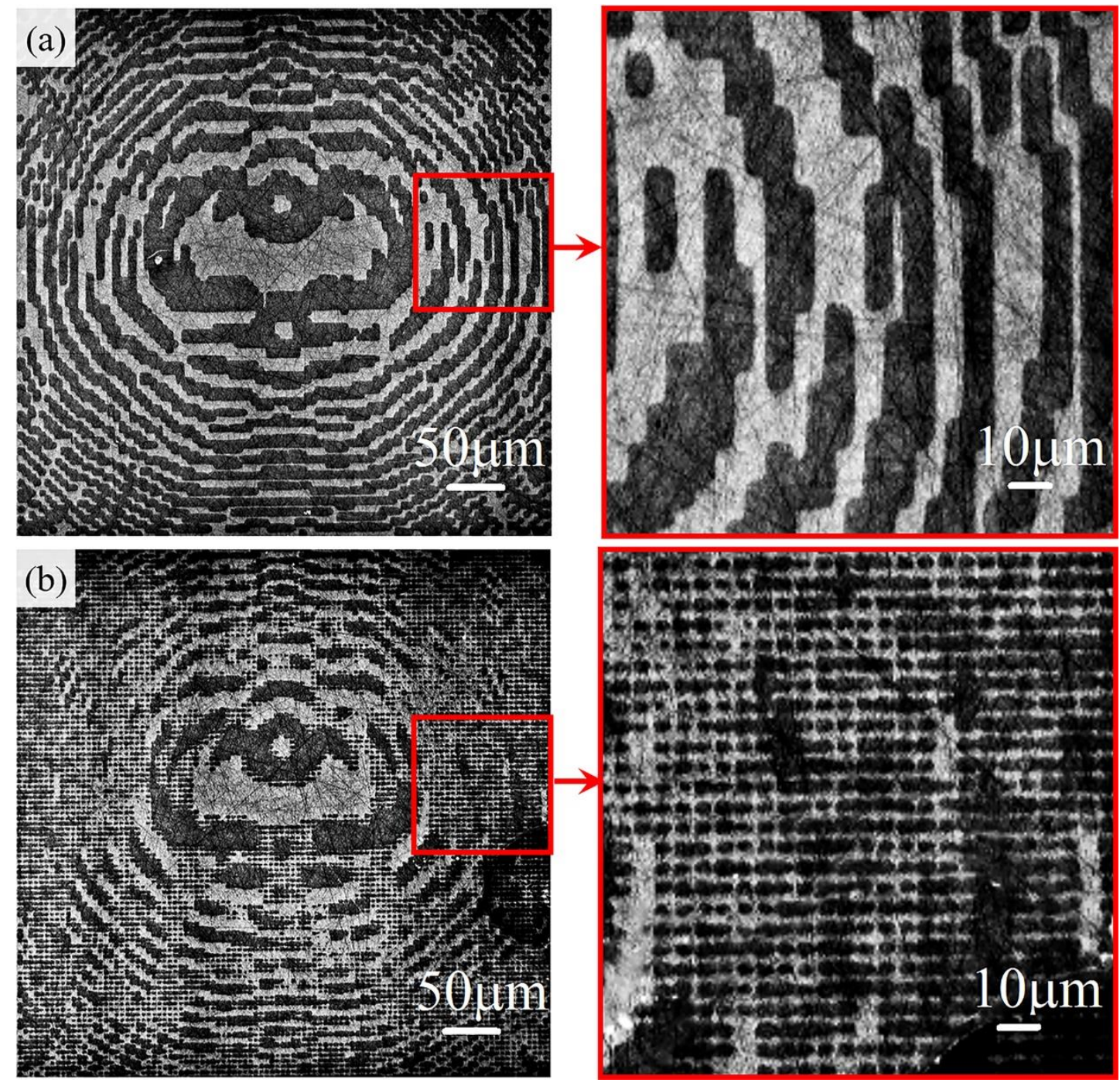

Figure S2. ESEM images of NPC structures designed by (a) the binary encoding method and (b) the QME method.

\section{Section III: Methods for nonlinear efficiency enhancement}

Further improvement can be attained at lower input power by using phase matching techniques in the longitudinal direction. Perfect phase matching can be achieved by selecting bulk materials with a larger coherence length $\left(l_{c}=\pi / \Delta k\right)$ to 
realize high-efficiency nonlinear beam shaping and imaging, seen in Figure S3a. Here, $\Delta k$ represents the phase mismatches between the FW and the SHW. Quasi-phasematching (QPM) techniques can be achieved by periodically flipping different regions of the structures (at a coherence length distance), seen in Figure S3b. QPM techniques can be realized by three-dimensional nonlinear photonic crystals. In 3D case, the transverse and longitudinal phase matching conditions can be satisfied simultaneously to enhance the nonlinear efficiency.

The QME method proposed in our manuscript demonstrates experimentally twodimensional NPCs for high-quality imaging and gives motivation for three-dimensional NPCs, QPM and additional manipulations of nonlinear interactions.
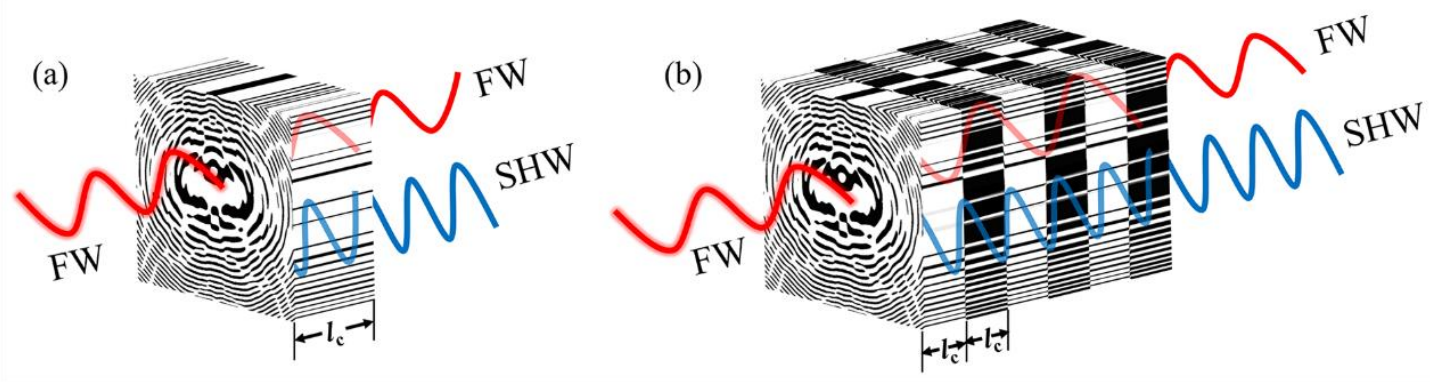

Figure S3. Illustration of different phase matching configurations with bulk nonlinear materials. (a) Perfect phase matching with uniform structures; (b) QPM with NPCs. 